
Sociale, 2014, p. 125-163.

\title{
L’horizon de la violence asymbolique dans la pensée de Pierre Bourdieu
}

\author{
Igor Krtolica
}

L'œuvre de Pierre Bourdieu témoigne d'une étrange unité. Car il se pourrait bien que ses travaux les plus anciens soient aussi les plus actuels, que l'analyse du sous-prolétariat algérien de la fin des années 1950 anticipe celle des travailleurs précaires et des chômeurs à l'ère du capitalisme néolibéral. "Travail et travailleurs en Algérie, mon livre le plus ancien et, peut-être, le plus actuel $» . .{ }^{1}$ Cet après-coup de Bourdieu sur son œuvre ne retrace pas une lente évolution, comme si une unité s'était progressivement constituée au fil des livres et venait se révéler à la fin ; il fait plutôt résonner la fin avec le commencement, comme si un souterrain les reliait secrètement ou qu'un pont avait été jeté par-dessus l'abîme qui les sépare. Un tel rapprochement s'explique pourtant aisément, pourvu que l'on tienne compte du déplacement historique qui, selon Bourdieu, le sous-tend: la précarité qui frappait conjoncturellement les sous-prolétaires algériens serait devenue une réalité structurelle des sociétés capitalistes contemporaines, le tableau des algériens d'hier se présentant alors rétrospectivement comme le portrait anticipé de l'insécurité généralisée des travailleurs d'aujourd'hui. «La précarité est aujourd'hui partout» - tel est le diagnostic qui redonne leur actualité à des travaux vieux de presque quarante ans.

C'est à tort que l'on prend parfois la sociologie bourdieusienne pour une réflexion sur les mécanismes de reproduction sociale. Ses premiers travaux sur l'Algérie présentent des analyses de crise ou de mutation: passage d'une économie précapitaliste à une économie capitaliste, crise de l'agriculture traditionnelle algérienne ${ }^{2}$. Or ce sont ces travaux qui sont à l'origine de la théorie de la pratique. Il est vrai que celle-ci implique une certaine circularité dialectique, dans la mesure où les structures sociales objectives sont intériorisées dans des dispositions subjectives puis reproduites par elles; le sens pratique ne serait alors rien d'autre que cet ajustement préréflexif des habitus aux structures. Il n'en reste pas moins que, de l'aveu de Bourdieu, cette conception n'est pas née de l'analyse de la reproduction sociale, à l'œuvre par exemple dans le champ scolaire, mais des enquêtes algériennes sur la crise de l'économie précapitaliste, où se manifeste au contraire un profond désajustement des habitus et des structures sociales. "Ce n'est pas par hasard, écrit-il en 1976 dans la préface d'Algérie 60 , si l'interrogation sur les relations entre les structures et les habitus s'est constituée à propos d'une situation historique où elle se proposait en quelque sorte dans la réalité même, sous la forme d'une discordance permanente entre les dispositions économiques des agents et le monde économique dans lequel ils devaient agir $»^{3}$.

La notion de violence symbolique incarne la circularité dialectique entre les structures sociales et les habitus, ce double mouvement d'intériorisation des contraintes objectives dans des dispositions subjectives (l'habitus comme structure structurée) et de reproduction de ces

\footnotetext{
${ }^{1}$ P. BourdiEU, «La précarité est aujourd'hui partout » (1997), Contre-feux, Paris, Raisons d'agir, 1998, p. 97.

${ }^{2}$ P. Bourdieu, Sociologie de l'Algérie, Paris, PUF, 1958 ; P. BOURDIEU, Travail et travailleurs en Algérie, Paris-La Haye, Mouton, 1963 (repris et synthétisé dans Algérie 60, Paris, Minuit, 1977) ; P. BouRDIEU, A. SAYAD, Le déracinement, Paris, Minuit, 1964.

${ }^{3}$ P. BOURDIEU, Algérie 60. Structures économiques et structures temporelles, p. 7.
} 
mêmes contraintes par la pratique (l'habitus comme structure structurante). Coextensive à toute la théorie de la pratique, une telle violence constitue le moteur principal de la reproduction sociale et de la domination, leur vecteur le plus puissant, le plus immédiat et le plus méconnu. Bourdieu estime en ce sens que «l'analyse de l'acceptation doxique du monde, en raison de l'accord immédiat des structures objectives et des structures cognitives, est le véritable fondement d'une théorie réaliste de la domination et de la politique ${ }^{4}$. Or, si la violence symbolique repose sur cet ajustement des dispositions subjectives aux structures sociales et si elle s'exerce d'autant mieux qu'elle est méconnue par les agents qui en sont le siège et le renfort, il est manifeste qu'un tel ajustement fait défaut aux sous-prolétaires algériens, incapables de conformer leurs dispositions aux exigences sociales nouvelles qui leur sont imposées, privés du sens pratique que cet ajustement rendait auparavant possible. Par un tour étrange, il faut donc admettre que leur impuissance radicale les conduit à se soustraire au jeu d'une violence symbolique qu'ils ne relaient plus. Non pas qu'ils échappent à la violence en général, loin de là, mais plutôt que la violence symbolique s'efface désormais devant une autre forme de violence. Tout se passe alors comme si, dans l'œuvre de Bourdieu, la scène algérienne constituait une limite paradoxale, le point d'apparition et de disparition de la théorie de la pratique et de la violence symbolique, l'indice d'une autre scène et d'une autre violence. Et s'il est vrai que la situation des travailleurs algériens au seuil des années 1960 devient la réalité structurelle des sociétés capitalistes occidentales, ne faut-il pas dire que c'est toute la sociologie de Bourdieu, tant du point de vue de l'analyse de la pratique sociale effective que du point de vue de sa théorisation, qui est aspirée par cette limite paradoxale ?

\section{Le cas algérien et le devenir réel des catégories abstraites de l'économie}

On sait que, s'ils se recoupent en bien des points, Algérie 60 et Le déracinement n'ont pas le même objet. Algérie 60 analyse la transformation corrélative des structures économiques et des structures temporelles. Bourdieu y montre comment l'émergence d'une économie capitaliste modifie en profondeur le rapport des agents sociaux au temps, substituant à la visée d'un avenir "virtuellement enfermé dans le présent directement perçu », visée propre au temps cyclique de la production précapitaliste, la représentation d'un futur « comme champ de possibles qu'il appartient au calcul d'explorer et de maîtriser ${ }^{5}$. S'introduit alors une discordance profonde entre les espérances subjectives des individus et les chances objectives qui leur sont offertes, entre leurs dispositions temporelles liées à leur expérience du temps cyclique de la reproduction économique simple d'un côté et les contraintes économiques nouvelles fondées dans le procès de production capitaliste et sa reproduction élargie de l'autre. Le déracinement examine pour son compte la crise de l'agriculture traditionnelle algérienne en fonction des déplacements et regroupements massifs imposés aux populations rurales, pendant la guerre, par le pouvoir colonial. Bourdieu et Sayad y montrent alors comment l'arrachement de populations entières à leurs terres et la mise en contact forcé d'individus d'âges et de milieux sociaux différents provoquent une transformation violente et irrémédiable, ainsi qu'une homogénéisation brutale de leurs modes d'existence.

Dans ces deux études, la situation coloniale agit pour Bourdieu comme un accélérateur des transformations sociales ${ }^{6}$. Algérie 60 décrit en effet l'importation à marche forcée, par le régime colonial, d'une organisation économique exogène. Rosa Luxemburg

\footnotetext{
${ }^{4}$ P. Bourdieu, L. WacQuant, Réponses. Pour une anthropologie réflexive, Paris, Seuil, 1992, p. 143.

${ }^{5}$ Ibid., p. 19.

${ }^{6}$ Cf. ibid., p. 13-14; voir aussi, P. BOURDIEU, A. SAYAD, Le Déracinement. La crise de l'agriculture traditionnelle en Algérie, p. 35, p. 99.
} 
avait montré que le processus d'accumulation primitive n'a pas seulement lieu aux origines du mode de production capitaliste mais qu'il est constamment rejoué, à chacune de ses phases d'expansion, comme une condition interne de sa dynamique : ainsi de la phase d'expansion impérialiste, qui réveille ainsi, sous de nouvelles conditions, le capitalisme sauvage des origines $^{7}$. De même chez Bourdieu, le phénomène colonial se présente comme un processus brutal de destruction des structures précapitalistes existantes et d'imposition de nouvelles structures adaptées aux exigences capitalistes. Nous avons dit que Le Déracinement donne à voir, à travers les déplacements systématiques des populations rurales imposés par le pouvoir français, la même mutation exogène et accélérée des structures économiques et cognitives de la paysannerie. Or, pour Bourdieu, ces analyses complètent celles d'Algérie 60 parce qu'elles montrent comment le déracinement, qui détruit les cadres spatiaux et temporels de l'existence ordinaire, achève ce que la généralisation des échanges monétaires avait commencé. Cet effet d' "accélération pathologique » des transformations sociales a pour l'historien ou le sociologue une valeur éminemment heuristique. Il donne à voir, au moment même où il est en train de disparaître, la nature de l'espace social ${ }^{8}$. Par les crises qu'elle provoque, l'accélération de l'histoire rend donc visible, au moment où elle se rompt, la dialectique des structures sociales et des habitus.

Mais qu'est-ce qui définit une situation de crise? Sur la base de cas précis de regroupements de populations, Le Déracinement décrit un phénomène de « dépaysannisation », c'est-à-dire les mutations que l'exode rural imprime à l'agriculture traditionnelle (disparition du rapport du fellah à la terre et aux bêtes, émergence du travail et généralisation des échanges monétaires) ainsi qu'à l'ensemble des valeurs traditionnelles de la société paysanne (rapports de prestige et poids de la hiérarchie familiale patriarcale, code de l'honneur, etc.). Algérie 60, dont le spectre est plus large puisqu'il prend en compte, à l'échelle de l'Algérie tout entière, l'économie capitaliste sous ses différents aspects, montre l'imposition accélérée et exogène de nouvelles données économiques (émergence de l'idée de propriété privée, du rapport entre activité productive et argent sous la forme du travail salarié), dont la fonction n'est plus sociale mais devient d'abord économique (non plus remplir ses obligations envers le groupe, mais gagner de l'argent à proportion du travail effectué, c'est-à-dire faire un travail rentable). Mais dans les deux cas, ce qui définit une situation de crise, ce n'est pas seulement le caractère accéléré de la transformation sociale, ni même l'effet de déculturation brutale, mais d'abord et surtout la coexistence des contraires au sein d'une même formation sociale: coexistence de l'ancien et du nouveau dans les structures objectives de l'économie comme dans les dispositions subjectives des agents, pris dans des cadres de références incompatibles (double bind $)^{9}$. «Aujourd'hui, c'est tout un peuple qui, incertain de sa démarche, va hésitant et trébuchant. La logique même de la situation coloniale a fait surgir un nouveau type d'hommes, qui se laissent définir négativement, par ce qu'ils ne sont plus et par ce qu'ils ne sont pas encore, les paysans

\footnotetext{
${ }^{7}$ Cf. R. LuXemburg, L'accumulation du capital [1913], Paris, Maspéro, 1967.

${ }^{8}$ On ne croira pas, pourtant, que l'accélération de l'histoire n'a pas transformé la nature du processus historique. Car il n'y a pas une simple différence de degré mais une profonde différence de nature entre un changement culturel progressif relativement endogène et un changement culturel brutal imposé de l'extérieur. Cf. P. BOURDIEU, A. SAYAD, Le Déracinement, p. 35 : «ceux qui argueraient que cette politique n'a fait que précipiter un mouvement qui se fût en tout cas accompli, indépendamment de toute intervention extérieure, ont pour eux toutes les apparences : de fait, entre autres facteurs, l'implantation en Algérie d'une économie capitaliste, la généralisation des échanges monétaires, la contagion culturelle favorisée par l'émigration et enfin la scolarisation ont pu déterminer, même dans les régions qui n'ont pas connu la colonisation agraire, une transformation généralisée des comportements, des attitudes et de l'ethos qui en était solidaire. Mais en réalité, la simple accélération qu'on lui fait subir, suffit à transformer le changement culturel dans sa nature même. »

${ }^{9}$ Sur cet effet de double-bind produisant des « habitus déchirés », cf. le chapitre II d'Algérie 60.
} 
dépaysannés, êtres auto-destructifs qui portent en eux-mêmes tous les contraires ${ }^{10}$. Chez Bourdieu, la coexistence des contraires au sein d'une même formation sociale se manifeste par un double effet de non-contemporanéité, comme si la société était à la fois en retard et en avance sur elle-même : non-contemporanéité des structures sociales et non-contemporanéité des habitus ${ }^{11}$.

La crise ne se définit pas seulement par ces deux contradictions. Car il n'y a pas seulement contradiction simultanée au niveau des structures économiques (entre une organisation précapitaliste et une organisation capitaliste) et au niveau des dispositions subjectives et des idéologies (entre des habitus correspondant à chacune des deux formes économiques). Il y a également un décalage entre les premières et les secondes, dans la mesure où les habitus se transforment plus lentement que les structures. On sait que Bourdieu nomme effet d'hystérésis cette inertie des dispositions subjectives, des schèmes de perception, de pensée et d'action, qui les font survivre dans un milieu qui a disparu. Le Déracinement en donne de nombreux exemples, ainsi des vieux paysans algériens qui suscitent ironie et mépris parce qu'ils valorisent à outrance le code traditionnel de l'honneur quand les jeunes gens sont parvenus à s'adapter à la nouvelle donne sociale et que la société tout entière est désormais soumise à la rentabilité des activités. «Parce qu'elles ne se transforment pas au même rythme que les structures économiques, des dispositions et des idéologies correspondant à des structures économiques différentes, encore actuelles ou déjà abolies, coexistent dans la société et parfois même à l'intérieur des individus $»^{12}$. La conséquence principale de cette triple discordance (à l'intérieur des structures, à l'intérieur des dispositions et entre les deux) est que les habitus devenus dysfonctionnels conduisent à la mise en suspens de l'adhésion au monde, comme si les agents ne parvenaient plus à le rencontrer ${ }^{13}$. Revenant à la fin des années 1990 sur ses travaux algériens, Bourdieu écrira : "Dans les situations de crise ou de changement brutal, celles notamment qui s'observent à l'occasion des contacts de civilisation liés à la situation coloniale ou à des déplacements très rapides dans l'espace social, les agents ont souvent peine à tenir ensemble les dispositions associées à des états ou à des étapes différentes et certains d'entre eux, souvent ceux qui, précisément, étaient les mieux adaptés à l'état antérieur du jeu, ont peine à s'ajuster au nouvel ordre établi : leurs dispositions deviennent dysfonctionnelles et les efforts qu'ils peuvent faire pour les perpétuer contribuent à les enfoncer plus profondément dans l'échec. [...] L'habitus a ses ratés, ses moments critiques de déconcertement et de décalage: la relation d'adaptation immédiate est suspendue $»^{14}$.

Revenant en 1977 sur les résultats de Travail et travailleurs en Algérie, Bourdieu regrette de n'avoir pas eu l'occasion d'affiner ses analyses en fonction de sa théorie de la pratique élaborée dans l'intervalle ${ }^{15}$. Il est donc instructif d'observer comment, vingt ans plus tard, dans les Méditations pascaliennes, Bourdieu fait à nouveau retour sur ses textes algériens pour en évaluer le sens et la portée. Car l'intérêt d'ensemble de ces après-coups n'est pas seulement qu'ils rappellent la valeur heuristique des situations critiques (qui révèlent

\footnotetext{
${ }^{10}$ P. Bourdieu, A. SAYAd, Le Déracinement, p. 161.

${ }^{11}$ P. BourdieU, Algérie 60, p. 15 : «tant au niveau des structures économiques que des dispositions, des représentations et des valeurs, on observe la même dualité, comme si ces sociétés n'étaient pas contemporaines d'elles-mêmes ».

${ }^{12}$ Ibid., p. 15. C'est un aspect que marque bien Bruno Karsenti lorsqu'il insiste sur la distinction entre deux durées ou deux rythmes historiques irréductibles, l'un qui relève d'une histoire de la production, l'autre qui relève d'une histoire des producteurs (cf. B. KARSENTI, « De Marx à Bourdieu. La critique et le dilemme de la pratique », in M. de Fornel, A. OGIEN (éd.), Bourdieu. Théoricien de la pratique, Paris, EHESS, 2011, p. 125129).

${ }^{13}$ Cf. ibid., p. 67.

${ }^{14}$ P. Bourdieu, Méditations pascaliennes [1997], Paris, Seuil, 2003, p. 232-233.

${ }^{15}$ Cf. P. Bourdieu, Algérie 60, Préface.
} 
la nature du sens pratique au moment où disparaît l'ajustement ordinaire des habitus aux structures sociales), mais surtout qu'ils théorisent un type d'expérience sociale négative (voire le négatif de toute expérience sociale), dont le sens réside dans un certain passage à la limite dans la théorie de la pratique, et dont la portée est étendue à l'ensemble de la situation actuelle. - Un type d'expérience sociale négative, car les sous-prolétaires algériens sont désormais assimilés à la catégorie générale des «hommes sans avenir » qui, dépourvus de toute fonction sociale, désajustés au monde existant, et ayant du même coup perdu le sentiment d'un rapport d'anticipation à un monde tangible, sont devenus incapables de s'y projeter ${ }^{16}$. "Privés de cet univers objectif d'incitations et d'indications qui orientent et stimulent l'action et, par là, toute la vie sociale, ils ne peuvent vivre le temps libre qui leur est laissé que comme temps mort, temps pour rien, vidé de tout sens. Si le temps semble s'anéantir, c'est que le travail salarié est le support, sinon le principe, de la plupart des attentes, des exigences, des espérances et des investissements dans le présent, ainsi que dans l'avenir ou le passé qu'il implique $\gg^{17}$. Dans le discours bourdieusien, la catégorie d'homme sans avenir vient donc d'abord qualifier, au niveau des modes de temporalisation, la figure économique bien connue du sous-prolétaire. - Chez Bourdieu, cette expérience sociale négative n'a pas le sens d'une promesse révolutionnaire. Si le sous-prolétariat de masse n'est pas l'incarnation d'une nouvelle puissance révolutionnaire, c'est qu'il n'y a pour Bourdieu nul travail du négatif, nulle contradiction motrice. On ne sera donc pas trompé par l'oscillation constante des sous-prolétaires entre la résignation et l'utopie, entre l'à-quoi-bon et l'attente eschatologique, entre le désespoir et le messianisme. Celle-ci exprime plutôt leur manque de prise sur la réalité objective, un sentiment profond de perte en monde. Dès 1964, en pleine période de décolonisation et de ferveur révolutionnaire tiers-mondiste, Bourdieu mettait déjà en garde : «il faut tout ignorer de la condition des ouvriers agricoles et des paysans dépaysannés, hantés par l'incertitude du lendemain, empêchés de trouver dans un monde qui les écrase un début de réalisation de leurs espérances, et n'ayant d'autre liberté que d'exprimer leur révolte par la tricherie avec l'effort et par la ruse quotidienne qui ronge peu à peu le sentiment de la dignité, pour accorder quelque créance aux prophéties eschatologiques qui voient en la paysannerie des pays colonisés la seule classe véritablement révolutionnaire ${ }^{18}$. Avant d'être l'amorce d'un hypothétique devenir-révolutionnaire, la condition $\mathrm{du}$ sous-prolétaire est donc l'expérience d'une impossible pratique de transformation sociale. Il y a certes, dans toute structure sociale, différents degrés de désajustement entre les chances objectives offertes par les structures socio-économiques et les espérances subjectives qu'un agent donné peut légitimement nourrir ; mais il y a surtout un seuil en deçà duquel aucune pratique n'est plus possible, expérience durable d'impuissance ${ }^{19}$. Et Bourdieu n'aura de cesse d'insister sur ce seuil d'effondrement de la pratique, dont l'impossible temporalisation vers l'avenir est le signe infaillible: «Les conduites souvent désordonnées, voire incohérentes, et sans cesse contredites par le discours, de ces hommes sans avenir, livrés aux aléas de ce qui leur advient au jour le jour et voués à l'alternance de l'onirisme et de la démission, de la fuite dans l'imaginaire et de la soumission fataliste aux verdicts du donné, attestent que, en deçà d'un certain seuil de chances objectives, la disposition stratégique elle-même, qui suppose la référence pratique à un à venir, parfois très éloigné, $[\ldots]$ ne peut se constituer $»^{20}$. - La portée de cette expérience sociale négative doit

\footnotetext{
${ }^{16}$ Cf. ibid., p. 318-322 : «Une expérience sociale : des hommes sans avenir ».

${ }^{17}$ Ibid., p. 320.

${ }^{18}$ P. Bourdieu, A. SAYAD, Le Déracinement, p. 170.

${ }^{19}$ Cf. P. Bourdieu, Algérie 60, chap. III : «Espérances subjectives et chances objectives ». Voir également, P. BOURDIEU, « La fabrique de l'habitus économique » [2003], in Esquisses algériennes, Paris, Seuil, 2008, p. 249250.

${ }^{20}$ P. BOURDIEU, Méditations pascaliennes, p. 319.
} 
être mesurée à l'extension générale que lui donne Bourdieu au cours des années 1990. Les analyses de cette période, inaugurée par La misère $d u$ monde, avancent une hypothèse récurrente: l'expérience des hommes sans avenir se serait généralisée, le désajustement radical des chances et des espérances ne serait plus lié à une situation locale et conjoncturelle de crise mais à une évolution générale et structurelle. Ainsi, à propos des sociétés occidentales : "C'en est fini à jamais de ces univers où la coïncidence quasi parfaite des tendances objectives et des attentes faisait de l'expérience du monde un enchaînement continu d'anticipations confirmées. Le manque d'avenir, qui était réservé jusque-là aux "damnés de la terre", est une expérience de plus en plus répandue, sinon modale $»^{21}$. Nous reconnaissons là le diagnostic qui fait pour Bourdieu l'étrange actualité de ses travaux sur l'Algérie, qui fonde l'analogie entre les prolétaires et sous-prolétaires algériens de la fin des années 1950 d'un côté et les travailleurs précaires et les chômeurs français des années 1990 de l'autre. «Les chômeurs et les travailleurs précaires, parce qu'ils sont atteints dans leur capacité de se projeter dans l'avenir, qui est la condition de toutes les conduites dites rationnelles, à commencer par le calcul économique, ou, dans un tout autre ordre, l'organisation politique, ne sont guère mobilisables. Paradoxalement, comme je l'ai montré dans Travail et travailleurs en Algérie, mon livre le plus ancien et, peut-être, le plus actuel, pour concevoir un projet révolutionnaire, c'est-à-dire une ambition raisonnée de transformer le présent par une référence à un avenir projeté, il faut avoir un minimum de prise sur le présent. Le prolétaire, à la différence du sous-prolétaire, a ce minimum d'assurances présentes, de sécurité, qui est nécessaire pour concevoir l'ambition de changer le présent en fonction de l'avenir escompté. Mais, soit dit en passant, il est aussi quelqu'un qui a quelque chose à défendre, quelque chose à perdre, son emploi, même épuisant et mal payé, et nombre de ses conduites, parfois décrites comme trop prudentes, ou même conservatrices, ont pour principe la crainte de tomber plus bas, de redescendre dans le sous-prolétariat $\gg^{22}$.

On trouve dans les Méditations pascaliennes, au milieu de développements sur les modes de temporalisation et la pratique, une surprenante interprétation du Procès de Kafka. Bourdieu y compare les victimes des violences extrêmes du $\mathrm{XX}^{\mathrm{e}}$ siècle, le personnage de $\mathrm{K}$. et la figure du sous-prolétaire. «Dans les situations extrêmes où l'incertitude et l'investissement sont simultanément portés à leur maximum, parce que, comme dans un régime despotique ou un camp de concentration, il n'y a plus de limites à l'arbitraire et à l'imprévisibilité, tous les enjeux ultimes, y compris la vie et la mort, se trouvent engagés à tout instant : chacun y est livré sans défense (comme K. ou comme les sous-prolétaires) aux formes les plus brutales de manipulation des craintes et des attentes $\gg^{23}$. À partir de l'impuissance à se temporaliser vers l'avenir, Bourdieu suggère un rapprochement entre des populations soumises à des politiques exceptionnelles (violences totalitaires, expériences des camps) et à une dépossession économique extrême. On ne croira pourtant pas que l'originalité de la comparaison se trouve là. Bourdieu n'est pas le premier à risquer une analogie entre ces deux limites de la domination, à suggérer que la figure du sous-prolétaire réveille le spectre des victimes des camps. Son originalité nous semble venir ici de la figure de K., utilisée comme moyen terme de l'analogie: car la figure idéal-typique de K. témoigne d'un véritable devenir-réel de la figure abstraite et fantasmée de l'homme sans avenir, déterminé comme sous-prolétaire. La tendance qui soumet les individus au procès de production comme pure force de travail n'est pas sans rappeler le devenir pratiquement vrai des catégories abstraites de l'économie politique, que Marx considérait comme le trait essentiel des sociétés capitalistes les plus modernes $^{24}$. Une telle hypothèse se trouve confirmée par Bourdieu dans le texte clôturant

\footnotetext{
${ }^{21}$ Ibid., p. 313, p. 336.

${ }^{22}$ P. BOURDIEU, Contre-feux, p. 97.

${ }^{23}$ P. BourdieU, Méditations pascaliennes, p. 331.

${ }^{24} \mathrm{Cf}$. K. MARX, Introduction à la critique de l'économie politique [1857], in K. MARX, Philosophie, Paris,
} 
Contre-feux, «Le néo-libéralisme, utopie (en voie de réalisation) d'une exploitation sans limite ». À propos de la conception néo-libérale de l'économie, Bourdieu y affirme en effet : «Cette théorie tutélaire est une pure fiction mathématique, fondée, dès l'origine, sur une formidable abstraction [...]. Cela dit, cette "théorie" originairement désocialisée et déshistoricisée a, aujourd'hui plus que jamais les moyens de se rendre vraie, empiriquement vérifiable $»^{25}$. Autre manière de dire que l'histoire tend à identifier la logique des choses aux choses de la logique.

La limite qui sépare le prolétariat du sous-prolétariat possède une valeur décisive, tant du point de vue pratique que du point de vue épistémologique. Elle marque en effet à la fois le seuil d'effondrement de la pratique et l'incapacité de la théorie à concevoir une telle expérience autrement que négativement, sous les catégories de l'impuissance, de la dépossession ou de l'absence d'avenir. Or, si l'on se rappelle le rôle constitutif que l'étude de la société algérienne a joué dans l'élaboration de la théorie de la pratique, cette limite semble toucher au cœur de la sociologie bourdieusienne, en constituer l'objet propre et pourtant inappropriable, l'horizon ou le foyer. C'est cette limite qui sépare le prolétariat du sousprolétariat que nous devons considérer maintenant, en fonction de son origine objective (l'économie capitaliste) et de ses conséquences subjectives (effondrement de la pratique).

\section{Les sociétés précapitalistes, l’État et le capitalisme}

Les études initiales sur l'Algérie décrivent le passage d'une économie précapitaliste à une économie capitaliste ; celles, tardives, sur l'ordre néolibéral examinent l'imposition d'un nouveau mode de domination économique au sein des sociétés du capitalisme avancé. De ces deux séries de textes, il serait insuffisant de dire que le capitalisme constitue le dénominateur commun - si l'on ne précisait immédiatement : le capitalisme dans sa tendance immanente à se reproduire à une échelle sans cesse élargie. À travers le cas algérien, Bourdieu retrouve l'un des enjeux majeurs du matérialisme historique, l'analyse des stades du capitalisme. Distinguons-en quatre : un premier stade, au cours duquel le capitalisme originel se forme en Europe occidentale sur les ruines de la société féodale, qui débute avec la phase d'accumulation primitive au $\mathrm{XV}^{\mathrm{e}}$ et $\mathrm{XVI}^{\mathrm{e}}$ siècles et inaugure le passage à des sociétés mercantiles (soumission du pouvoir des villes qui s'était constitué dans les pores des monarchies féodales, concentration du pouvoir dans des régimes absolutistes exerçant leur souveraineté sur le marché intérieur et le commerce extérieur, développement du commerce triangualaire); un deuxième stade, qui commence avec la révolution industrielle à la fin du XVIII $^{\mathrm{e}}$ siècle et débouche au siècle suivant sur un formidable mouvement d'expansion impérialiste et coloniale (crise des monarchies européennes, constitution des États-nations et de leurs Empires coloniaux, exode massif en Amérique du Nord) ; un troisième, qu'amorce la mondialisation du capitalisme au moment de la Seconde Guerre mondiale et que poursuit la décolonisation et l'essor du Tiers monde (mondialisation de l'économie, instauration d'un

Gallimard, 1982, p. 475-476 : «L'indifférence à l'égard du travail particulier correspond à une forme de société dans laquelle les individus passent avec facilité d'un travail à un autre, et dans laquelle le genre déterminé du travail leur paraît fortuit et par conséquent indifférent. Le travail est alors devenu, non seulement en tant que catégorie, mais dans la réalité même, un moyen de produire la richesse en général, et il a cessé de se confondre avec l'individu en tant que destination particulière de celui-ci. Cet état de choses s'est le mieux développé dans le type le plus moderne de la société bourgeoise, aux Etats-Unis. C'est là que la catégorie abstraite, "travail", "travail en général", travail sans phrase, le point de départ de l'économie moderne, devient pratiquement vrai. Ainsi l'abstraction la plus simple que l'économie moderne place au premier rang et qui exprime un phénomène ancestral, valable pour toutes les formes de société n'apparaît pourtant comme pratiquement vraie, dans cette abstraction, qu'en tant que catégorie de la société la plus moderne » (tr. fr. M. Rubel et L. Évrard).

${ }^{25}$ P. Bourdieu, Contre-feux, p. 108-109. 
rapport économique de dépendance inégale entre le Nord et le Sud, élargissement de l'Étatprovidence dans les pays du capitalisme avancé) ; un quatrième enfin, qui voit s'effectuer le démantèlement progressif de l'État national-social en Occident à partir de la crise des années $1970^{26}$, et conduit à l'imposition graduelle de l'ordre économique néolibéral comme nouveau mode de subsomption de la force de travail au procès de production (émergence d'un chômage structurel instaurant un nouveau mode d'exploitation fondé sur la précarisation généralisée des travailleurs, intériorisation du rapport de dépendance inégale entre le centre et la périphérie au sein des pays du capitalisme avancé et apparition de tiers mondes intérieurs $)^{27}$. Dans ce cadre général, un des intérêts des études algériennes de Bourdieu est qu'elles montrent en accéléré et comme à l'état pur le mécanisme présidant à la transition d'une société précapitaliste fondée sur la reproduction simple vers une société capitaliste fondée sur la reproduction élargie, c'est-à-dire qu'elle grossit les effets destructeurs du capitalisme sur les structures sociales et les habitus. De ce point de vue, Bruno Karsenti a raison de considérer que la sociologie bourdieusienne est polarisée par l'opposition entre les sociétés archaïques précapitalistes et les sociétés du capitalisme avancé ${ }^{28}$. Ces deux types de société constituent en effet deux espaces sociaux inverses, le processus social régissant les unes se présentant comme la reproduction d'unités fragmentaires quasiment indifférenciées et le processus social gouvernant les autres comme un mouvement de détotalisation et de dédifférenciation. Entre ces deux pôles, cependant, surgit l'État.

Dans l'œuvre de Bourdieu, la question de l'État n'intervient pour elle-même que tardivement, après la crise économique des années 1970 et l'avènement du néolibéralisme, alors que le démantèlement de l'État national-social a déjà été largement amorcé. Reste que l'État s'est toujours présenté chez lui comme une réalité socio-politique centrale et ambiguë ${ }^{29}$. Réalité ambiguë d'abord, dans la mesure où, pour Bourdieu, l'État ne se réduit ni à un simple appareil répressif au service des dominants ni à une instance neutre d'arbitrage et de résorption des conflits, mais participe un peu des deux. Tel est d'ailleurs le principe de l'appréciation ambivalente de l'institution scolaire dans Les héritiers puis dans La reproduction. L'école y apparaît à la fois comme le lieu où s'apprend l'exercice de la raison et se propagent les valeurs culturelles légitimes, moyen d'accès à l'universel et de réduction des inégalités, et comme le milieu de reproduction de ces mêmes inégalités, reproduction d'autant plus performante qu'elle est relayée par l'adhésion des agents aux valeurs méritocratiques (violence symbolique). Or, à l'instar de l'école, tous les champs relevant de la sphère publique de l'État apparaissent sous ce double visage: comme un espace de diffusion publique de l'universel et comme l'instance participant du monopole de la violence symbolique légitime ${ }^{30}$. Réalité centrale ensuite, dans la mesure où l'État est l'archétype de toute structure sociale. On sait, au moins depuis la publication des cours sur l'État, que cette question s'inscrit chez Bourdieu dans le cadre d'une théorie générale de l'espace social, et plus particulièrement d'une réflexion sur la genèse et la structure des champs de pouvoir. Et si

\footnotetext{
${ }^{26}$ Nous empruntons à Étienne Balibar l'expression suggestive d'État national-social qui désigne simultanément l'État-nation et l'État-providence. Voir entre autres, E. BALIBAR, Nous, citoyens d'Europe?, Paris, La Découverte, 2001, passim.

${ }^{27}$ Sur cet aspect décisif historiquement et théoriquement, cf. l'introduction et le post-scriptum à P. BOURDIEU, Les structures sociales de l'économie, Paris, Seuil, 2000. Bourdieu y analyse les conditions d'apparition de l'État et distingue les différentes phases de son évolution en rapport avec l'évolution du champ économique.

${ }^{28}$ Cf. B. KARSENTI, «De Marx à Bourdieu. La critique et le dilemme de la pratique », in M. de ForNEL, A. OGIEN (éd.), Bourdieu. Théoricien de la pratique, Paris, EHESS, 2011, p. 128-131.

${ }^{29}$ Sur l'évolution des références à la notion d'État dans le discours bourdieusien, nous reprenons les éléments dégagés dans la «Situation du cours » de P. BourdiEU, Sur l'État. Cours au Collège de France (1989-1992), Paris, Seuil, 2011, p. 594-601.

${ }^{30}$ Voir par exemple, P. BouRdiEU, « Espace social et pouvoir symbolique », in Choses dites, Paris, Minuit, 1987, p. 163, ou bien P. BOURDIEU, L. WACQUANT, Réponses. Pour une anthropologie réflexive, p. 87.
} 
un champ se définit au sens strict comme une sphère de production relativement autonome dans l'espace social, l'État doit être considéré comme un «méta-champ » où l'on lutte pour déterminer la position que les différents champs (économique, académique, artistique, journalistique, etc.) peuvent respectivement occuper. L'État incarne le champ par excellence, produit d'un double mouvement de différenciation progressive (différenciation des sociétés en champs relativement autonomes) et de concentration du pouvoir (monopolisation du pouvoir au sein d'un champ politique supérieur aux autres champs). En tant que totalité interne différenciée en champs distincts, l'État occupe une position intermédiaire entre les sociétés précapitalistes d'une part, unités fragmentaires quasi indifférenciées où des champs ne peuvent se constituer faute d'une division sociale du travail suffisante, et la formation capitaliste d'autre part, processus tendanciel de détotalisation et de dédifférenciation de l'ensemble des champs et des structures sociales auxquels elle impose ses contraintes économiques.

Bourdieu ne manque pas d'observer que cette double tendance qui a présidé à la constitution de l'État s'essouffle et s'inverse à partir des années 1980. En témoigne la disparition croissante des champs : les sphères de production autonomes se trouvent de plus en plus fortement soumises aux exigences d'une économie marchande sur laquelle l'État semble incapable de peser. De ce point de vue, comme l'a montré Cyril Lemieux, le travail de Bourdieu sur la télévision et le journalisme dans les années 1990 peut être lu comme une analyse de la dilution progressive d'un champ de pouvoir dans l'espace de l'économie capitaliste $^{31}$. Et à cette époque, ce sont plus généralement les appels répétés de Bourdieu au maintien des protections sociales, sa défense obstinée des institutions publiques qui peuvent être interprétés en fonction de cette tendance, dans laquelle Bourdieu fait valoir le rôle historique de l'État, entre la disparition des sociétés archaïques et l'essor du capitalisme néolibéral. On sait en effet que, d'une main, l'État affranchit les individus des liens de dépendance qui, dans les sociétés précapitalistes, bridaient les possibilités d'ascension sociale et les moyens de renverser de la domination (cf. le rôle de l'institution scolaire), tandis que de l'autre il oppose avec ses protections sociales un frein sérieux aux contraintes économiques du capitalisme ${ }^{32}$. On ne s'étonnera donc pas que, si Bourdieu ne cache pas une forme de nostalgie à l'égard des sociétés précapitalistes, qui maintiennent une harmonie entre les habitus et les structures sociales (par exemple les sociétés traditionnelles algériennes), ce n'est pourtant jamais elles qu'il invoque comme moyen de contrecarrer les effets destructeurs du néolibéralisme. Ce rôle est explicitement et exclusivement dévolu à l'État ${ }^{33}$. Toutefois, en raison de la subordination tendancielle du politique à l'économique, Bourdieu fait moins appel au réveil de l'État national qu'à la constitution d'un État supranational ou transnational, seule instance politique à même de contrebalancer à l'échelle mondiale les contraintes économiques néolibérales. La construction d'un État européen se présente alors à

\footnotetext{
${ }^{31}$ Sur la disparition progressive des champs en fonction de l'évolution du capitalisme, voir le bel article de C. LEMIEUX, «Le crépuscule des champs. Limite d'un concept ou disparition d'une réalité historique ? », in M. de Fornel, A. Ogien (éd.), Bourdieu. Théoricien de la pratique, p. 92-93. Sur le champ journalistique, cf. P. BOURDIEU, Sur la télévision, Paris, Liber, 1996.

${ }^{32}$ L'œuvre de sociologie du travail de Robert Castel fut profondément habitée par la recherche d'un équilibre entre l'émancipation à l'égard des formes de dépendance sociale et professionnelle d'un côté et la protection contre les effets dévastateurs du capitalisme de l'autre. Cf. R. CASTEL, Métamorphoses de la condition sociale. Une chronique du salariat, Paris, Fayard, 1995 ; Propriété privée, propriété sociale, propriété de soi (avec Claudine Haroche), Paris, Fayard, 2001 ; L’insécurité sociale. Qu'est-ce qu'être protégé ?, Paris, La République des idées / Seuil, 1993 ; La discrimination négative. Citoyens ou indigènes ?, Paris, La République des idées / Seuil, 2007.

${ }^{33}$ Nous ne pouvons donc suivre les conclusions de Bruno Karsenti sur ce point, qui nous paraissent minorer le rôle décisif que Bourdieu entend faire jouer à l'État à partir des années 1990 (cf. B. KARSENTI, « De Marx à Bourdieu. La critique et le dilemme de la pratique », in M. de Fornel, A. OgIEn (éd.), Bourdieu. Théoricien de la pratique, p. 129-133.
} 
ses yeux comme « une étape sur le chemin de l'État universel $»^{34}$.

Comme le signalait pourtant l'analyse de l'institution scolaire, nulle référence n'est chez lui plus ambiguë que la référence à l'universel. Car l'universel n'est pas moins un vecteur d'émancipation raisonnable pour les dominés qu'un masque de la violence symbolique des dominants. Il est vrai que cette ambiguïté change de sens dès lors qu'on parvient à l'exploiter. Tel est d'ailleurs l'objet de la «Realpolitik de l'universel », par laquelle Bourdieu entend promouvoir une nouvelle Aufklärung. Il ne s'agit plus seulement de la promotion abstraite et naïvement optimiste de l'universel, mais également d'une «lutte permanente pour l'universalisation des conditions d'accès l'universel », lutte qui redouble le projet kantien en contribuant pratiquement à la sortie de l'homme hors de l'état de minorité et au progrès de l'État universel dans l'histoire ${ }^{35}$. Ce combat pour l'universel au sein des champs et institutions qui s'en réclament (l'école et l'université, la justice, et surtout l'État) ne consiste pas à les mettre en face de leur propre contradiction (puisqu'elles promeuvent l'universel sans fournir à tous les moyens d'y accéder et de le produire); il consiste à exploiter l'ambiguïté de l'intérêt qu'elles lui accordent pour faire réellement progresser l'universel. Pour Bourdieu, «Si l'universel avance, c'est parce qu'il existe des microcosmes sociaux qui, en dépit de leur ambiguïté intrinsèque, liée à leur enfermement dans le privilège et l'égoïsme satisfait d'une séparation statutaire, sont le lieu de luttes qui ont pour enjeu l'universel et dans lesquelles des agents ayant, à des degrés différents selon leur position et leur trajectoire, un intérêt particulier à l'universel, à la raison, à la vérité, à la vertu, s'engagent avec des armes qui ne sont autre chose que les conquêtes les plus universelles des luttes antérieures $»^{36}$.

Peut-être l'universel avance-t-il, avec tout son cortège d'ambiguïtés. Mais l'État en est-il le seul vecteur? Loin s'en faut. Bourdieu montre au contraire que le passage des formations sociales archaïques à l'État, et de l'État aux formations capitalistes, implique chaque fois deux choses : l'apparition d'un degré d'universalisation supérieur et l'instauration d'un nouveau mode de domination. Nous avons vu que le processus d'accumulation, de concentration et d'unification qui préside à l'apparition et au maintien de l'État sur les débris des sociétés pré-étatiques constitue, d'une part, un ensemble de mécanismes «qui contribuent à porter l'ensemble des processus sociaux concernés à un degré d'universalisation supérieur en les arrachant aux particularismes (linguistiques, culturels, mais aussi économiques) associés à la particularité du local », mais que cette universalisation n'empêche pourtant pas, d'autre part, que «l'unification profite aux dominants» qui dissimulent le monopole de la violence symbolique derrière un État présenté comme instance impartiale d'arbitrage ${ }^{37}$. Or, il faut dire de même que le processus qui préside à l'unification du champ économique mondial implique un degré d'universalisation supérieur par rapport à l'État et l'instauration d'un nouveau mode de domination. L'analogie entre État et capitalisme est donc double. Car, en premier lieu, pas plus que l'État n'entraînait la dissolution pure et simple des espaces sociaux pré-étatiques, la formation d'un champ économique mondial, qui déborde pourtant de tous côtés les cadres imposés par l'État national (notamment dans le domaine financier), n'entraîne une disparition de l'État lui-même (qui se trouve néanmoins dépossédé des possibilités politiques d'arbitrage et de régulation, réduit à la surveillance des fluctuations de la Bourse). Et si Bourdieu rejette l'idée que le champ mondial de l'économie serait homogène, puisqu'il se présente plutôt comme un ensemble de sous-champs mondiaux polarisé autour de certaines économies nationales, l'unification économique libérale mondiale n'en porte pas moins l'ensemble des processus sociaux à un degré d'universalité supérieur à celui de l'État. D'où

\footnotetext{
${ }^{34}$ P. BouRdieu, Contre-feux, p. 17 ; cf. Contre-feux 2, Paris, Raisons d'agir, 2001, passim.

${ }^{35}$ P. Bourdieu, Méditations pascaliennes, p. 121. Cf. ibid., p. 116.

${ }^{36}$ Ibid., p. 177-178.

${ }^{37}$ P. BOURDIEU, Les structures sociales de l'économie, p. 274.
} 
l'analogie possible, en second lieu, entre les deux mécanismes d'unification et de monopolisation du pouvoir : «En fait, l'intégration au champ économique mondial par le libre-échange, la libre-circulation du capital et la croissance orientée vers l'exportation qui est proposée aux pays dominés comme un destin ou comme un idéal (par opposition à une orientation plus nationaliste cherchant à développer la production domestique pour le marché intérieur) présente la même ambiguité que l'intégration au champ économique national en d'autres temps : tout en donnant toutes les apparences d'un universalisme sans limites, elle sert les dominants, c'est-à-dire les grands investisseurs qui, tout en se situant au-dessus des États, et en particulier le plus puissant d'entre eux politiquement et militairement, les EtatsUnis, pour assurer les conditions favorables à la conduite de leurs activités économiques $»^{38}$. Ce processus d'unification économique mondial se fait donc ici aussi au service des dominants. Ce sont en effet «la logique du champ et la force propre du capital concentré qui imposent des rapports de force favorables aux intérêts des dominants », quoique leur domination soit dissimulée derrière des institutions internationales d'arbitrage prétendument neutres (FMI, OMC) $)^{39}$.

Or, si le degré d'universalisation du capitalisme est supérieur à celui de l'État (puisqu'il est sans limites) et si l'universalisme n'est pas plus ambigu dans un cas que dans l'autre (puisque l'unification se fait chaque fois au service des dominants), on voit mal en quoi l'universalisme ambigu du capitalisme ne pourrait pas être exploité aussi bien que celui de l'État universel. Pourquoi des deux universalisations, l'une politique et l'autre économique, Bourdieu est-il conduit à privilégier la première et à négliger la seconde ${ }^{40}$ ? Pourquoi l'État universel, qui implique une monopolisation mondiale de la violence symbolique légitime, serait-il plus désirable que le capitalisme? Et l'on voit encore moins pourquoi l'on n'exploiterait pas les tendances historiques qui y conduisent déjà, là où celles qui pourraient mener à l'État universel sont loin d'être mobilisées. Derechef, qu'est-ce qui justifie que l'émergence de forces politiques menant à l'État universel soient plus désirables que l'essor des forces économiques mondiales du capitalisme? Sur ce point délicat, Bourdieu ne prend pas la peine de répondre clairement, comme s'il allait de soi qu'un ordre économique mondial ne pouvait être ni désirable ni même désiré. La raison nous semble formulée en creux dans son discours : le capitalisme est impossible à désirer parce que sa dynamique immanente implique, objectivement, la dissolution tendancielle de toutes les structures sociales et, subjectivement, le franchissement du seuil d'effondrement de la pratique. Dans sa tendance développée, le capitalisme serait donc le négatif de toute formation sociale.

\section{De la pratique à la théorie}

\footnotetext{
${ }^{38}$ Ibid., p. 278 (nous soulignons).

${ }^{39}$ Ibid., p. 280. Sur les deux aspects de l'analogie, unification et domination, voir l'entretien récemment paru : P. BOURDIEU, « Mondialisation et domination : de la finance à la culture », Cités, 2012, n 51, p. 129-130.

${ }^{40} \mathrm{Il}$ est clair du moins pour Bourdieu que l'unification économique du monde ne mènera pas d'elle-même à son unification politique : si le capitalisme s'appuie sur l'intervention de l'État pour se développer, sa tendance immanente ne le porte jamais à se soumettre à une instance extra-économique, au pouvoir juridico-politique de l'État. Peut-être faut-il s'attendre en revanche à ce que les effets de l'économie capitaliste favorisent indirectement l'émergence d'un État supranational. Cf. P. BouRdiEU, Les structures sociales de l'économie, p. 25, p. 280 : «Il est vain d'espérer que l'unification conduise par sa seule logique à une véritable universalisation, assumée par un État universel. Mais il n'est sans doute pas déraisonnable d'attendre que les effets de la politique d'une petite oligarchie attentive à ses seuls intérêts économiques à court terme puissent aussi favoriser l'émergence progressive de forces politiques, elles aussi mondiales, capables d'imposer peu à peu la création d'instances transnationales chargées de contrôler les forces économiques dominantes et de les subordonner à des fins réellement universelles ».
} 
Il semble que, pour Bourdieu, le type de violence propre au capitalisme ne soit pas convertible dans une Realpolitik de l'universel. Nous demandons si, toutefois, la mise en échec de la pratique, effet négatif provoqué par la violence du capitalisme néolibéral, ne serait pas compensée par un effet positif, à savoir par une prise de conscience critique des mécanismes de la domination. Nous savons que, pour s'exercer, la violence symbolique suppose de la part des agents qui la supportent et la relaient une méconnaissance « fondée sur l'ajustement inconscient des structures subjectives aux structures objectives ${ }^{41}$. Or, s'il existe une corrélation entre l'efficacité de la violence symbolique et le degré d'ajustement des dispositions aux structures sociales, et si pour espérer s'en affranchir, les individus doivent prendre conscience du mécanisme par lequel ils contribuent à la reproduction de l'ordre social, une telle conscience n'est-elle pas favorisée par le désajustement radical ?

La sociologie a pour Bourdieu une vocation éthique et politique: en tant que connaissance réflexive des conditions sociales de la pratique, elle réduit notre méconnaissance des mécanismes sociaux qui nous déterminent et nous permet de nous en affranchir partiellement ${ }^{42}$. D'agents sociaux passivement soumis à des causalités sociales méconnues, la réflexivité du savoir sociologique fait de nous des sujets relativement libres à leur égard. Par conséquent, si la reproduction de la domination n'opère pleinement qu'à la faveur de la méconnaissance à laquelle la contrainte sociale doit une part de son efficacité, à l'inverse, la possibilité de se soustraire à l'efficace de la violence symbolique suppose une connaissance réflexive de ces contraintes, connaissance que procure notamment la sociologie. On sait comment Rancière dénonce l'opération: au philosophe-roi Bourdieu aurait substitué le sociologue-roi, et sous couvert de démystification aurait reconduit le point de vue dominant du maître (le discours qui dévoile la nécessité des mécanismes de la domination révèle en même temps «la nécessité de la méconnaissance par le dominé de la loi de cette domination $\gg)^{43}$. Telle est l'objection désormais classique que l'on oppose, sous diverses formes, à la vocation éthique et politique de la sociologie bourdieusienne. Il n'est pas d'autre manière d'y répondre que de comprendre comment s'acquiert effectivement cette connaissance réflexive que le sociologue-roi aurait confisquée à son profit. Remarquons au préalable qu'il n'arrive jamais à Bourdieu d'opposer frontalement la méconnaissance ou l'inconscience des uns, agents immergés dans la pratique, à la réflexivité ou à la conscience des autres, sociologues dégagés des urgences de l'action. C'est même l'originalité revendiquée de sa conception du sens pratique, qui repose sur la critique conjointe de l'objectivisme et du subjectivisme, renvoie dos-à-dos la «physique sociale » objectiviste suivant laquelle les agents sociaux seraient les objets passifs de mécanismes inconscients qui les dépassent (cf. le structuralisme de Lévi-Strauss et d'Althusser), et la "phénoménologie sociale » subjectiviste qui considère l'ordre social comme le produit des décisions et des actions maîtrisées d'individus conscients (idée qu'on retrouve, mutatis mutandis, aussi bien chez Sartre qu'en ethnométhodologie ou dans les théories du choix rationnel). La conception dispositionnelle et stratégique du sens pratique, d'après laquelle les schèmes de perception, de pensée et d'action sont d'une part le produit d'une intériorisation dans un ensemble de dispositions subjectives des exigences portées par les structures sociales objectives, et d'autre part des principes préréflexifs d'improvisations pratiques adaptées au jeu social, a donc pour intérêt premier d'échapper à la fausse alternative de l'action individuelle consciente et libre et de la soumission inconsciente aux lois sociales. En ce sens, si Bourdieu peut affirmer après

\footnotetext{
${ }^{41}$ P. Bourdieu, L. WACQuAnt, Réponses. Pour une anthropologie réflexive, p. 142.

${ }^{42}$ Cf. ibid., p. 171.

${ }^{43}$ J. RANCIERE, Et tant pis pour les gens fatigués, Paris, Éditions Amsterdam, 2009, p. 134. Sur la critique du sociologue-roi, voir par ailleurs J. RANCIERE, Le philosophe et ses pauvres [1983], Paris, Flammarion, 2007, p. 239-288.
} 
Leibniz que les trois quarts de nos actions sont empiriques, c'est que le plus souvent nous ne réfléchissons pas les principes qui nous déterminent à agir, mais que nous ne sommes nullement voués à les méconnaître. À l'évocation par Roger Chartier de l' «image stéréotypée de [son] travail, qui est pensé comme mettant à nu des contraintes contre lesquelles on ne pourrait rien faire et qui broient les individus en ne leur donnant aucune place », Bourdieu rétorque ainsi : "nous naissons déterminés et nous avons une petite chance de finir libres: nous naissons dans l'impensé et nous avons une toute petite chance de devenir des sujets. [...] On ne naît pas le sujet de ses pensées, on en devient le sujet, à condition, entre autres choses [...] de se réapproprier la connaissance des déterminismes ${ }^{44}$. La conception du sens pratique laisse donc ouverte en droit la possibilité d'un passage de la méconnaissance à la connaissance réflexive, de la passivité à l'activité, de la servitude à la liberté.

La question reste néanmoins de savoir à quelles conditions cette possibilité est susceptible de se réaliser. Or Bourdieu observe que, à la faveur d'un désajustement entre les habitus et les structures sociales, les individus accèdent bien à une forme de réflexivité. Chaque fois que les habitus viennent à dysfonctionner (en raison d'un effet d'hysteresis, d'un déplacement rapide de l'agent dans l'espace social, ou encore d'un décrochage radical par rapport au jeu social), l'individu est conduit à prendre conscience de la dialectique qui gouvernait auparavant sa pratique, et qui demeurait inaperçue tant qu'elle fonctionnait harmonieusement. Au fil des ans, Bourdieu ne manquera d'ailleurs guère de remarquer que l'ajustement entre les habitus et les structures sociales n'est qu'un cas particulier qu'il faut se garder d'universaliser. Un tel ajustement est, à l'échelle individuelle, le cas le plus fréquent. «L'ajustement immédiat entre l'habitus et le champ n'est qu'une des formes possibles de l'action, même si c'est la plus fréquente : "Nous sommes empiriques disait Leibniz — par quoi il entendait pratiques -, dans les trois quarts de nos actions" ${ }^{45}$. À l'échelle individuelle, l'adaptation du sens pratique aux structures sociales est en effet la règle ordinaire, qu'illustre à merveille le cas des sociétés archaïques précapitalistes. Tel n'est pourtant pas le cas à l'échelle de l'espace social, même dans les sociétés archaïques. C'est pourquoi il arrive à Bourdieu de dire que l'ajustement est un cas-limite jamais incarné en fait : «l'homologie entre l'espace des positions et l'espace des dispositions n'est jamais parfaite $»^{46}$. Ce jeu dans la structure, dû en partie au décalage intergénérationnel, est la condition de possibilité de la mobilité au sein de l'espace social, et de sa transformation souvent insensible. Ces deux remarques de principe, qui ne se contredisent nullement mais se complètent en fonction de l'échelle d'analyse, valent indifféremment pour toute structure sociale. En revanche, lorsque Bourdieu affirme que l'ajustement des habitus aux structures sociales est un cas de plus en plus rare, que «c'en est fini à jamais de ces univers où la coïncidence quasi parfaite des tendances objectives et des attentes faisait de l'expérience du monde un enchaînement continu d'anticipations confirmées », nous n'avons plus affaire à une remarque de principe, mais à un diagnostic historique sur la tendance générale des sociétés contemporaines $^{47}$. Or, si ces expériences sociales de désajustement se multiplient, et si elles conduisent à une prise de conscience réflexive des conditions sociales de la domination symbolique, n'y a-t-il pas là une chance pour la théorie critique? On pourrait à bon droit le penser. Pourtant, Bourdieu signale que la réflexivité qui résulte quasi mécaniquement du désajustement est une réflexivité pratique qui, quant au seuil d'objectivation requis par la réflexivité critique, reste insuffisamment libérée du besoin d'agir : " la relation d'adaptation immédiate est suspendue, dans un instant d'hésitation où peut s'insinuer une forme de

\footnotetext{
${ }^{44}$ P. Bourdieu, R. Chartier, Le sociologue et l'historien, Marseille, Agone \& Raisons d'agir, 2010, p. $39-41$.

${ }^{45}$ P. Bourdieu, L. WACQUANT, Réponses. Pour une anthropologie réflexive, p. 107.

${ }^{46}$ P. BOURDIEU, Méditations pascaliennes, p. 226.

${ }^{47}$ Ibid., p. 336.
} 
réflexion qui n'a rien à voir avec celle du penseur scolastique et qui, à travers les mouvements esquissés du corps [...] reste tournée vers la pratique et non vers celui qui l'accomplit ${ }^{48}$. On ne croira pas que ces considérations rejouent, au sein du discours sociologique, l'opposition philosophique de la contemplation et de l'action, de la pensée pure et de la pensée pratique. Car, d'après cette opposition, c'est seulement lorsque l'individu se soustrait aux sollicitations matérielles et à la nécessité d'agir qu'il s'ouvre à la pensée pure, et que celle-ci, comme le disait Hegel, rentre enfin chez soi (bei sich); alors que, pour Bourdieu, l'objectivation théorique et la réflexivité critique n'affranchissent jamais complètement le théoricien des conditions socioéconomiques, matérielles et empiriques qui rendent sa pensée possible. Pas plus que la contemplation du philosophe, la réflexivité critique du sociologue ne foule le domaine illusoire de la pensée pure.

Dans ces remarques, suivant les positions respectives des agents dans l'espace social, nous trouvons néanmoins le moyen de faire une typologie des rapports entre théorie et pratique chez Bourdieu, c'est-à-dire des possibilités concrètes de réfléchir et de s'affranchir des déterminations sociales qui structurent la pratique. Ici encore, la limite entre prolétariat et sous-prolétariat s'avère décisive. $-1^{\circ}$ En premier lieu, les agents sociaux qui occupent une position dominante dans un champ de pouvoir donné n'ont pas réellement l'occasion de prendre conscience de la dialectique des structures sociales et des habitus qui déterminent leur pratique, dans la mesure où ils possèdent des habitus adaptés au monde dans lequel ils évoluent. Il n'est pas sûr qu'ils aient d'ailleurs intérêt à en prendre conscience, dans la mesure où leur domination est d'autant plus efficace et paraît d'autant plus légitime qu'elle passe pour naturelle. $-2^{\circ}$ Les agents sociaux qui occupent une position dominée dans un champ auquel ils sont intégrés, à l'instar des travailleurs prolétaires, ont en revanche un intérêt objectif à prendre conscience des ressorts de la domination dont ils sont les victimes. Cependant, pour autant que leur travail les maintient dans le jeu social, ils possèdent une prise sur le présent et une faculté de se projeter raisonnablement dans l'avenir, mais leurs conduites restent souvent prudentes ou conservatrices par crainte du déclassement (que le chômage structurel rend aujourd'hui toujours probable), crainte qui constitue un puissant levier de démobilisation non seulement des individus sans qualification mais aussi de l'ensemble des travailleurs. «L'existence d'une armée de réserve, que l'on ne trouve plus seulement, du fait de la surproduction de diplômés, aux niveaux les plus bas de la compétence et de la qualification technique, contribue à donner à chaque travailleur le sentiment qu'il n'a rien d'irremplaçable et que son travail, son emploi est en quelque sorte un privilège, et un privilège fragile et menacé [...]. Cette sorte de "mentalité collective" [...], commune à toute l'époque, est au principe de la démoralisation et de la démobilisation que l'on peut observer (comme je l'ai fait dans les années 60, en Algérie) dans des pays sous-développés, affligés de taux de non-emploi ou de sous-emploi très élevés et habités en permanence par la hantise du chômage $»^{49}$. Nuls plus que ces travailleurs hantés par la peur du chômage et de la descente dans le sous-prolétariat ne sont donc aujourd'hui victimes de la violence symbolique, car leurs efforts pour rester partie prenante du jeu social les conduit à se faire les relais d'une domination dont ils sont les cibles privilégiées, comme en témoignent les employés prêts à renoncer à certains droits pour préserver leur emploi. - $3^{\circ} \mathrm{A}$ contrario, si le degré de désajustement des sous-prolétaires (chômeurs ou travailleurs précaires) aux structures sociales dont ils sont exclus leur fournit des occasions de réfléchir les ressorts de la domination symbolique, c'est-à-dire la complicité ordinaire entre les habitus et les structures objectives, et s'il leur rend en même temps évident l'intérêt qu'ils auraient à renverser la domination, leur exclusion hors du jeu social leur ôte cependant toute prise suffisante sur ses

\footnotetext{
${ }^{48}$ Ibid., p. 233.

${ }^{49}$ P. BOURDIEU, Contre-feux, p. $96-97$.
} 
mécanismes et, corrélativement, l'espoir raisonnable d'une action efficace pour l'avenir. Surtout que, dépossédés des moyens matériels de subvenir à leur existence, les sousprolétaires sont pris dans une urgence pratique qui les empêche de réfléchir autrement que pratiquement les mécanismes de la domination dont ils sont les victimes, et donc de prendre la distance objective requise par la théorie. $-4^{\circ}$ Les ressources économiques et sociales qui permettent un tel niveau d'objectivation théorique, voilà précisément ce dont est muni l'intellectuel ou l'homo academicus. Si sa réflexivité est proprement théorique, c'est précisément que l'oisiveté de la skholè dont il jouit l'affranchit des nécessités de l'action qui maintenaient la prise de conscience des autres individus à un niveau encore pratique. Toutefois, si l'on admet que le degré de méconnaissance des déterminations sociales varie avec l'ajustement de l'habitus au champ, et si l'on admet que l'occupation d'une position dominante dans un champ de pouvoir donné favorise la tendance à défendre sa propre position, le statut de l'intellectuel pose deux problèmes. Ces problèmes concernent tout particulièrement le sociologue qui se fait fort de réfléchir et de critiquer les mécanismes de la violence symbolique. Car à la faveur de quelle expérience sociale l'intellectuel en vient à réfléchir de manière critique la dialectique qui soutient la pratique, lui qui ne semble pas soumis à l'insécurité du monde dans lequel il évolue? En outre, quel intérêt le porte initialement à désirer avec les autres et dans d'autres champs que le sien la critique de la domination ou son renversement, lui qui n'en est apparemment pas une victime particulièrement privilégiée?

Il ne suffira pas de dire que le métier d'intellectuel conduit naturellement à l'engagement politique, comme si l'un entraînait nécessairement l'autre. Car la théorie et la critique ne sont chez Bourdieu jamais intrinsèquement liées, pas même en sociologie, et surtout pas dans les autres champs de savoir (cf. le cas exemplaire de la philosophie) ${ }^{50}$. On ne se contentera pas non plus de l'idée que certains théoriciens sont conduits à réfléchir la domination des autres parce qu'ils sont eux-mêmes dominés dans le champ intellectuel. Car, au-delà du fait que le parcours intellectuel de Bourdieu le dément (de ses premiers travaux jusqu'à ses positions politiques de 1995), l'appartenance de l'intellectuel au champ académique implique une lutte autour du capital spécifique pour lequel il lutte dans son champ mais pas nécessairement autour des capitaux pour lesquels d'autres agents luttent dans d'autres champs. Il ne suffira pas, enfin, de montrer que l'intellectuel est un homme pluriel, évoluant dans un espace social complexe constitué de plusieurs champs dans lesquels il occupe une position chaque fois différente, ses habitus étant toujours partiellement désajustés aux structures sociales, de sorte qu'il ne peut jamais totalement méconnaître les déterminismes objectifs qui structurent ses propres dispositions ${ }^{51}$. Car cette conscience, même

\footnotetext{
${ }^{50}$ Cf. P. BourdiEU, Méditations pascaliennes, Introduction.

${ }^{51}$ Il nous semble que c'est le sens général des critiques que Bernard Lahire adresse à Bourdieu, tant du point de vue de la théorie des champs que de la théorie de l'habitus : la pluralité essentielle des structures objectives et subjectives, la diversité des champs dans lesquels un agent est amené à évoluer et des habitus qu'il est donc conduit à contracter et à mobiliser, constituent autant de possibilités de former un discours distancié par rapport à leur propre pratique. Voir B. LAHIRE, « Champ, hors-champ, contrechamp » et « De la théorie de l'habitus à une sociologie psychologique », in B. LAHIRE (dir.), Le travail sociologique de Pierre Bourdieu. Dettes et critiques, Paris, La Découverte \& Syros, 1999/2001, p. 23-57 et p. 121-152 ; voir également B. LAHIRE, L'homme pluriel : les ressorts de l'action, Paris, Nathan, 1998. À ce sujet, un des cas les plus intéressant dans l'œuvre de Bourdieu reste à nos yeux celui de ce cuisinier kabyle d'Alger, dont le sociologue entendit les propos en 1962, et qu'il qualifia par la suite d' «économiste spontané »- très rare cas où la barrière qui sépare la réflexivité critique de la réflexivité pratique se trouve brouillée dans le discours bourdieusien. "Cet homme doté d'une petite éducation élémentaire disait, avec ses mots, français ou berbères - à propos des choses de la tradition -, l'essentiel de ce que j'avais pu découvrir par ailleurs au prix d'un long travail de déchiffrement [...]. À la manière d'un économiste spontané, ce cuisinier proposait en quelques heures une vision globale, digne de la discussion scientifique, d'un univers sur lequel il avait pu prendre une vue à la fois approfondie et distanciée, du fait de la position qu'il occupait au sein de la société coloniale : position à la fois centrale - à la différence de la plupart
} 
vive, est une condition de possibilité de la réflexivité critique, mais non pas une condition suffisante. Et c'est bien, en dernière instance, le problème : si la distance par rapport aux urgences pratiques (skholè) est une condition de possibilité de la réflexivité critique, elle n'en est jamais la condition suffisante.

Tant dans ses analyses sociologiques positives et circonscrites que dans son travail d'épistémologue, Bourdieu ne s'est jamais départi d'un principe fondamentalement pragmatiste : nos facultés mentales ont avant tout un caractère pratique, elles sont d'abord tournées vers l'action. Et si la plupart de nos actions ne sont pas réfléchies, c'est justement que l'harmonie entre nos anticipations et la réalité ne nous y contraint pas. L'impuissance à agir (l'effondrement de la pratique) et le détachement par rapport aux nécessités pratiques (l'oisiveté scolastique) sont les seules situations capables de produire effectivement la conscience réflexive que tout agent peut en droit prendre sur les règles de sa propre pratique. Mais ces deux conditions sont-elles jamais réunies dans un même individu ? Il s'agit là de deux conditions très différentes, à la fois complémentaires et opposées, qui forment ensemble une sorte de chiasme. Dans la mesure même où elle est dégagée de la nécessité d'agir, la skholè est avec la maîtrise des outils symboliques une condition de possibilité générale de la réflexivité critique et de l'objectivation de la pratique - condition sine qua non, elle n'est pourtant pas une condition suffisante. À l'inverse, l'impuissance à agir est une condition réelle et singulière de la réflexivité, cause déterminante qui n'est toutefois pas non plus suffisante puisque la réflexivité demeure encore pratique, faute d'être affranchie des nécessités de l'action. Il existe donc deux conditions nécessaires de la réflexivité critique : l'une est une condition générale de possibilité, qui rend possible la connaissance critique en général sans y conduire nécessairement ; l'autre est une condition réelle et déterminante, qui contraint effectivement l'individu à réfléchir mais maintient sa réflexion à un niveau encore pratique. Il est requis que ces deux conditions, l'une socioéconomique et l'autre affective, soient réunies pour que n'importe quel agent social, y compris le sociologue, soit nécessairement conduit à prendre une attitude de réflexivité critique à l'égard des formes de la domination. Les deux conditions occupent donc chez Bourdieu des fonctions distinctes mais complémentaires : la skholè constitue le facteur abstrait qui rend possible la pensée critique en général sans la déterminer, et l'émotion le facteur concret qui l'oriente sur un objet déterminé. Ainsi arrive-t-il à Bourdieu de suggérer que le choix de faire porter l'analyse sociologique sur telle situation sociale plutôt que sur telle autre trouve sa raison déterminante non pas tant dans la fonction de travailleur intellectuel et dans les conditions socioéconomiques qu'elle suppose, que dans une émotion particulière qui anime la pensée du sociologue. Les motifs qui commandent et orientent la réflexion sociologique vers tel ou tel champ ont bien un caractère libidinal : pulsion, joie, colère, indignation, dégoût... ${ }^{52}$ Revenant sur son choix de travailler sur le bal des célibataires, il peut alors affirmer : " Je peux dire que j'ai mis près de vingt ans à savoir pourquoi j'avais choisi ce petit bal... Je crois même - ce sont des choses que je n'aurais jamais dites il y a encore dix ans - que l'émotion de sympathie - au sens fort - que j'ai éprouvée, le pathétique qui se dégageait de la scène que j'ai vue, etc.

des ouvriers et employés, il voyait le monde des Européens de l'intérieur - et, malgré tout, marginale, parce qu'il n'avait jamais rompu les liens avec tous les compagnons d'infortune qu'il avait côtoyés au cours d'une existence picaresque » (cf. P. BOURDIEU, «La fabrique de l'habitus économique » [2003], in Esquisses algériennes, p. 250-261).

${ }^{52}$ Cf. P. BOURDIEU, «Si le monde social m'est supportable, c'est parce que je peux m'indigner» [1989-1990], La Tour d'Aigues, Éditions de l'Aube, 2002, p. 51-57. En revanche, à notre connaissance, la question de savoir en quoi ces affects ont une valeur sociale, telle qu'on en vienne à désirer avec les autres et pour les autres leur libération, n'est pas résolue chez Bourdieu. Mais il est clair que ce rôle doit revenir à l'émotion, qui a toujours une portée collective et non pas subjective. À ce titre, «l'émotion de sympathie » dont parle Bourdieu ne doit pas être considérée comme une émotion parmi d'autres, mais comme une propriété structurelle de l'émotion. 
ont été au point de départ de l'intérêt que j'ai eu pour cet objet $»^{53}$.

Bien qu'elles soient toutes deux nécessaires, ces deux conditions sont empiriques. En dépit des apparences lexicales, la skholè n'est pas une condition de possibilité au sens transcendantal du terme. Elle est objective, historique, matérielle, sociale, économique, bref empirique, mais elle n'est pas transcendantale. Il en va de même de l'émotion qui déclenche et oriente l'attitude réflexive : elle est subjective, psychologique, individuelle, libidinale, empirique aussi bien. Quelle est alors chez Bourdieu la condition proprement transcendantale de la conscience réflexive en général ? La condition transcendantale de la réflexivité n'est ni le détachement par rapport aux nécessités de l'action, ni l'affect produit par une perception empirique, mais le retard originaire du sujet pratique par rapport au monde, c'est-à-dire le fait que la perception ne s'enchaîne pas immédiatement en action, mais que leur enchaînement soit disjoint par l'affection et la représentation ${ }^{54}$. Ce retard désigne moins l'inertie de fait des dispositions subjectives sur les structures sociales objectives (effet d'hysteresis), que l'écart en droit entre le sujet et la réalité matérielle qui l'affecte. C'est lui qui constitue la condition transcendantale des habitus préréflexifs et de la conscience réflexive, c'est-à-dire de la pratique en général. Or ce retard témoigne pour une tout autre mémoire et un tout autre inconscient que ceux qu'évoque le plus souvent Bourdieu. Non pas la mémoire corporelle des dispositions acquises, ni l'inconscience psychologique dans laquelle nous évoluons tant que nos actions s'insèrent harmonieusement dans le monde, mais une mémoire spirituelle et un inconscient métapsychologique qui conditionnent a priori l'intériorisation et la conservation des contraintes du monde social dans le corps et la conscience du sujet. Si la pratique témoigne de l'effort permanent de l'individu pour ajuster ses dispositions subjectives à la réalité objective, c'est qu'il y a un désajustement en droit, un retard constitutif du subjectif sur l'objectif. Il est vrai que le sens pratique parvient ordinairement à masquer l'étrange souvenir de ce retard. Mais chaque fois que se trouve franchi le seuil d'effondrement de la pratique, cet écart transcendantal jamais vécu, qui hante l'inconscient sous la forme d'un passé qui n'a jamais été présent, menace soudainement de submerger la conscience. Bourdieu ne pressent jamais autant l'éventualité d'une telle souffrance que lorsqu'il évoque, au plus près de Heidegger, comment l'émotion touche au cœur de l'ouverture affective de l'être au monde. "On pourrait, par un jeu de mot heideggerien, dire que la disposition est exposition. C'est parce que le corps est (à des degrés inégaux) exposé, mis en jeu, en danger dans le monde, affronté au risque de l'émotion, de la blessure, de la souffrance, parfois de la mort, donc obligé de prendre au sérieux le monde (et rien n'est plus sérieux que l'émotion, qui touche jusqu'au tréfonds des dispositifs organiques), qu'il est en mesure d'acquérir des dispositions qui sont elles-mêmes ouvertures au monde, c'est-à-dire aux structures mêmes du monde social dont elles sont la forme incorporée. [...] Nous apprenons par corps. L'ordre social s'inscrit dans les corps à travers cette confrontation permanente, plus ou moins dramatique, mais qui fait toujours une grande place à l'affectivité et, plus précisément, aux transactions affectives avec l'environnement social $»^{55}$. Reste que Bourdieu semble avoir évacué, dans son œuvre sociologique, le sens transcendantal et la nature métapsychologique de cette souffrance affective, en dépit de la direction que prenait son projet philosophique initial, inspiré de Heidegger. Avec l'abandon de ce projet à la fin des années 1950, la philosophie s'efface devant l'ethnologie, et l'analyse transcendantale des

\footnotetext{
${ }^{53}$ P. Bourdieu, L. WACQUAnt, Réponses. Pour une anthropologie réflexive, p. 139.

${ }^{54} \mathrm{Tel}$ est le sens transcendantal que Bruno Karsenti semble vouloir donner à l'hysteresis chez Bourdieu. Sur ce point délicat, on lira ses développements très suggestifs autour du problème du corps dans son article « De Marx à Bourdieu. Les dilemmes du structuralisme de la pratique ». Quoique Karsenti ne parle pas pour son compte de condition transcendantale, une telle instance nous semble bien être impliquée par le sens et la portée de ses analyses.

${ }^{55}$ P. Bourdieu, Méditations pascaliennes, p. 203-204.
} 
structures de la vie temporelle et affective cède sa place à une analyse empirique de l'expérience-limite du temps vide éprouvé par les sous-prolétaires algériens ${ }^{56}$.

Nous demandions si la violence extrême, en mettant en échec la pratique, ne pouvait conduire en contrepartie à une prise de conscience critique de la part des individus soumis à la domination. Il apparaît qu'une telle violence, non contente d'enrayer le sens pratique, ne permet pas non plus aux conditions empiriques de la réflexivité critique d'être réunies dans un même individu. De la sorte, ce n'est pas la pensée critique qui prend le relais d'une action devenue improbable, mais bien l'expérience affective d'un temps mort.

\section{La violence asymbolique du capitalisme et le problème de la vie affective}

Le problème général de la violence est constitutif de la pensée de Bourdieu parce qu'il se situe à l'articulation des structures objectives et subjectives. En ce sens, toute la théorie de la pratique et toutes les analyses positives l'enveloppent. La violence symbolique est une notion centrale de l'œuvre de Bourdieu en tant qu'elle exprime, théoriquement et pratiquement, la circularité dialectique des structures objectives et des dispositions subjectives. Circularité dialectique au sens où « la violence symbolique est, pour parler aussi simplement que possible, cette forme de violence qui s'exerce sur un agent social avec sa complicité $»^{57}$. Nous avons vu que cette complicité ou cette méconnaissance que suppose la violence symbolique pour s'exercer n'est jamais aussi forte que lorsque le monde va de soi ; que la violence symbolique n'exerce donc jamais aussi efficacement ses effets reproducteurs qu'au sein d'une sphère de production relativement autonome, c'est-à-dire au sein d'un champ social dans lequel les agents luttent pour la possession d'un capital spécifique assurant la domination à l'intérieur du champ, sans que la valeur de ce capital lui-même ne soit remise en question. C'est pourquoi, parodiant Weber, Bourdieu pouvait définir l'État, qui est le champ par excellence, par le monopole de la violence symbolique légitime. C'est pourquoi aussi l'institution scolaire, qui est un lieu de production des individus et de reproduction sociale, donne à observer au plus près les effets de cette violence symbolique (institution qu'Althusser pouvait alors appeler, pour des raisons similaires, "appareil idéologique d'État»). Sous tous ces aspects, la violence symbolique exprime les deux principes qui forment le cercle de la reproduction sociale : l'intériorisation de contraintes objectives et la reproduction des structures sociales existantes. En ce sens, elle témoigne pour Bourdieu d'une « loi de conservation de la violence », suivant laquelle la violence qui produit les agents se trouve réinjectée par eux dans l'espace social dont elle provient: " quand des gens subissent la violence, dans leur enfance, dans leur travail, etc., ils rendent la violence ». Dans tous les cas, « la violence, malheureusement, n'est jamais perdue $»^{58}$.

De ces deux caractères de la violence symbolique, intériorisation et reproduction, la situation existentielle des sous-prolétaires était la preuve a contrario. Les sous-prolétaires témoignent en effet d'une incapacité foncière à intérioriser dans des dispositions pratiques les exigences d'une structure sociale par rapport à laquelle ils ont décroché ; dès lors, disposant d'habitus inadaptés, échouant à s'insérer dans une structure sociale qui évolue sans eux, ils ne contribuent pas non plus à sa reproduction. Tel est le cas le plus flagrant d'un désajustement entre l'histoire de la production et l'histoire des producteurs. Ce cas-limite acquiert aujourd'hui une importance décisive puisqu'il devient un phénomène structurel, qui témoigne d'une violence capitaliste objective qui ne peut être ni intériorisée ni reproduite - c'est-àdire qui ne peut être dialectisée, mise en cercle. Car comment faire de l'incertitude et de

\footnotetext{
${ }^{56}$ Cf. ibid., p. 318-332.

${ }^{57}$ P. Bourdieu, L. WACQUANT, Réponses. Pour une anthropologie réflexive, p. 143.

${ }^{58} \mathrm{P}$. BOURDIEU, «Mondialisation et domination : de la finance à la culture », art. cit., p. 134.
} 
l'insécurité une anticipation raisonnable ? Et comment relayer une contrainte qu'on n'a même pas fait sienne? Cette violence capitaliste objective impossible à convertir dans des dispositions subjectives, à symboliser, doit donc être appelée violence extrême, inconvertible ou asymbolique. Sans la nommer spécifiquement, Bourdieu évoque à de nombreuses reprises cette autre forme de violence, cette «violence sans phrase », indiquant chaque fois qu'elle est fondée dans le stade actuel de la domination capitaliste, c'est-à-dire dans le nouveau procès d'accumulation qui réveille le « capitalisme sauvage des origines $»^{59}$.

Cette violence extrême inaugure pour Bourdieu un «mode de domination d'un type nouveau », fondé sur une double insécurité. Une insécurité objective, qui repose sur une précarisation et une mise en concurrence croissantes du travail à l'échelle mondiale et à tous les niveaux de la hiérarchie (flexibilisation des trajectoires professionnelles, invocation d'une concurrence généralisée hors de l'Europe et en Europe, hors de l'entreprise et au sein de l'entreprise), insécurité rendue possible par l'existence d'une armée de réserve de maind'œuvre soumise à la menace permanente du chômage ${ }^{60}$. L'insécurité objective de la «flexploitation»se redouble alors d'une insécurité subjective, qui se manifeste dans les habitus déchirés et précarisés des individus, soumis à la souffrance au travail et au stress, à la peur permanente du chômage et du sous-prolétariat, et dont ne préservent plus suffisamment les protections sociales de l'État en voie de démantèlement. La preuve de cette violence non dialectisable est donnée par la configuration spécifique prise par la loi de conservation de la violence : la violence n'y joue plus un rôle reproducteur. D'après Bourdieu, l'impossibilité d'intérioriser les contraintes insupportables infligées par l'économie capitaliste se manifeste par un retour différé de la violence subie (alcoolisme, suicides, petites et grandes violences quotidiennes, émeutes urbaines, terrorisme, etc.), dont le coût économique et social devient à son tour impossible à supporter par la structure sociale (insécurité des personnes et des biens, inflation des dispositifs de maintien de l'ordre, augmentation des demandes de protection sociale, etc. $)^{61}$. Le cercle de la violence symbolique s'est brisé, et avec lui l'équilibre de l'espace social.

En réalité, ce nouveau stade du capitalisme implique bien une forme de reproduction du système, non pas au sens de la reproduction simple des sociétés archaïques ou étatiques, mais au sens d'une reproduction élargie qui se confond avec la tendance immanente du capitalisme. Dans cette conservation de la violence, l'État joue un rôle paradoxal. Certes, il se présente comme le meilleur rempart contre la violence sauvage du nouveau procès d'accumulation, puisqu'il est encore en mesure d'offrir un minimum de protection sociale aux individus vivant sous la menace d'être réduits en pure force de travail ; mais ironiquement, parce qu'il atténue les effets de la violence néolibérale et préserve les citoyens d'une stricte domination économique, il favorise en retour la méconnaissance de cette violence. Avec l'affaiblissement progressif de l'État dans le champ économique mondial, la conservation de la violence prend donc un autre tour. Car la violence capitaliste, dont les effets sont de moins en moins atténués par l'État, ne constitue pas un vecteur de production subjective des agents (absence d'intériorisation de la violence et effondrement de la pratique) ni de reproduction objective de l'espace social (démantèlement de l'État, improductivité économique, politique et sociale sans cesse croissante), mais au contraire un vecteur de destruction et des individus et des structures sociales. L'opposition de la violence politique à la violence économique signifie que, pour Bourdieu, le capitalisme tend vers un seuil impolitique. Si la violence symbolique dominée par la figure de l'État est une notion centrale dans sa réflexion, il faut

\footnotetext{
${ }^{59}$ P. Bourdieu, Contre-feux, p. 99.

${ }^{60}$ Cf. ibid., p. 112, p. 99 : «Le fondement ultime de tout cet ordre économique placé sous l'invocation de la liberté des individus est en effet la violence structurale du chômage, de la précarité et de la peur qu'inspire la menace du licenciement ».

${ }^{61}$ Ibid., p. 46.
} 
dire alors que la violence asymbolique incarnée par le néolibéralisme en est en revanche la notion-limite.

Située au point d'articulation et de désarticulation des structures objectives et subjectives, la question de la violence asymbolique constitue la limite paradoxale de la réflexion bourdieusienne, l'au-delà de l'éthique et du politique. En témoignent deux indices récurrents dans l'œuvre : les dispositions temporelles et affectives des agents, et notamment l'expérience d'un temps mort comme seuil d'effondrement de la pratique; la limite entre prolétariat et sous-prolétariat dans l'espace social, traitée en fonction de la dynamique du capitalisme. Revenant sur ses enquêtes algériennes à trente ans de distance, Bourdieu déclare : «J'avais entrepris des recherches sur la "phénoménologie de la vie affective", ou plus exactement sur les structures temporelles de l'expérience affective. [...] Je me pensais comme philosophe et j'ai mis très longtemps à m'avouer que j'étais devenu ethnologue. [...] Je voulais par exemple établir le principe de la différence entre prolétariat et sous-prolétariat ; [...] j'ai essayé de montrer que le principe de cette différence se situe au niveau des conditions économiques de possibilité de prévision rationnelle, dont les aspirations révolutionnaires sont une dimension. [...] Je voulais aussi comprendre, à travers mes analyses de la conscience temporelle les conditions de l'acquisition de l'habitus économique "capitaliste" chez des gens formés dans un cosmos précapitaliste ${ }^{62}$. Nous croyons que le foyer ou l'horizon de l'étrange unité de l'œuvre de Pierre Bourdieu se trouve précisément dans le rapport entre les conditions politiques de l'action pratique et de la pensée critique et les contraintes économiques du capitalisme. Au foyer ou à l'horizon de ces problèmes se dresse toujours la violence asymbolique, violence sauvage et sans phrase, indice négatif d'un excès intraitable.

\footnotetext{
${ }^{62}$ P. BourdiEU, « Fieldwork in Philosophy », in Choses dites, p. 16-18.
} 